\title{
Prognosis of ocular myasthenia in Korea: A retrospective multicenter analysis of 202 patients
}

Yoon-Ho Hong a , Seok-Beom Kwon ${ }^{\text {b }}$, Byung-Jo Kim ${ }^{c}$, Byoung Joon Kim ${ }^{\text {d }}$, Seung Hyun Kim ${ }^{\text {e }}$, Jong Kuk Kim ${ }^{\mathrm{f}}$, Kyung-Seok Park ${ }^{g}$, Ki-Jong Park ${ }^{\mathrm{h}}$, Jung-Joon Sung ${ }^{\mathrm{i}}$, Eun Hee Sohn ${ }^{\mathrm{j}}$, Yeong-Bae Lee ${ }^{\mathrm{k}}$, Dushin Jeong ${ }^{\mathrm{l}}$, In Soo Joo ${ }^{\mathrm{m}, *}$, Byung-Ok Choi ${ }^{\mathrm{n}}$, Young-Chul Choi ${ }^{\circ}$ for the Korean Research Group for Neuromuscular Diseases

a Seoul National University Boramae Hospital, South Korea

${ }^{\mathrm{b}}$ Hallym University Hospital, South Korea

${ }^{\mathrm{c}}$ Korea University Medical Center, South Korea

${ }^{d}$ Sungkyunkwan University, Samsung Medical Center, South Korea

e Hanyang University Hospital, South Korea

${ }^{\mathrm{f}}$ Kosin University, Gospel Hospital, South Korea

${ }^{g}$ Seoul National University Bundang Hospital, South Korea

${ }^{\text {h }}$ Gyeongsang National University Hospital, South Korea

i Seoul National University Hospital, South Korea

j Chungnam National University Hospital, South Korea

${ }^{\mathrm{k}}$ Gachon University of Medicine and Science, South Korea

${ }^{1}$ Soonchunhyang University Cheonan Hospital, South Korea

${ }^{\mathrm{m}}$ Ajou University Hospital, South Korea

${ }^{\mathrm{n}}$ Ewha Womans University Dongdaemun Hospital, South Korea

- Yonsei University Youngdong Severance Hospital, South Korea

\section{A R T I C L E I N F O}

\section{Article history:}

Received 8 March 2008

Received in revised form 23 May 2008

Accepted 27 May 2008

Available online 3 July 2008

\section{Keywords:}

Ocular myasthenia gravis

Prognosis

AChR antibody

Thymoma

Repetitive nerve stimulation test

\begin{abstract}
A B S T R A C T
Objectives: The aims of this study were to obtain data on the frequency with which Korean patients with autoimmune myasthenia gravis (MG) present solely with ocular disturbances and progress to develop generalized disease and to identify the prognostic factors associated with secondary generalization.

Methods: We conducted a retrospective multicenter survey in which a total of 376 adult patients who were newly diagnosed with MG from 2000 through 2005 were reviewed for analysis. Patients with ocular MG at the time of symptom presentation $(n=202,53.7 \%)$ were divided into two subgroups according to their prognosis: the patients whose disease remained ocular throughout the follow-ups were placed in the OMG-R group, and the patients who progressed to develop generalized disease were placed in the OMG-G group. Clinical characteristics and laboratory findings were compared between the two subgroups.

Results: Secondary generalization developed in 47 (23.3\%) of the 202 study subjects, mostly within the first 6 months after symptom presentation, while the disease remained ocular throughout the follow-up duration (median 11.8 months) in the remaining 155 patients (76.7\%). AChR antibody, abnormal repetitive nerve stimulation tests (RNST) and thymoma were more frequently observed in the patients in the OMG-G group than in those in the OMG-R group ( $p<0.01$ in all). In seropositive cases, the titers of AChR antibody were also significantly higher in the OMG-G group than in the OMG-R group (median, $3.8 \mathrm{nM}$ vs. $6.4 \mathrm{nM} ; p<0.05$ ). Cox proportional hazards regression analyses showed that early oral prednisolone treatment significantly reduced the risk of secondary generalization ( $\mathrm{HR}, 0.24 ; 95 \% \mathrm{CI}, 0.11-0.56)$, whereas abnormal $\mathrm{AChR}$ antibody ( $\mathrm{HR}, 5.34 ; 95 \% \mathrm{CI}, 1.60-17.8$ ) and thymoma (HR, 2.32; 95\% CI, 1.21-4.45) were predictive of the development of secondary generalization.

Conclusions: Our findings suggest that several factors, including the AChR antibody, thymoma, early corticosteroid treatment, and possibly latent neuromuscular abnormality revealed by RNST, may have an impact on the risk of developing generalized disease in Korean patients presenting with ocular myasthenia. (c) 2008 Elsevier B.V. All rights reserved.
\end{abstract}

\footnotetext{
* Corresponding author. Department of Neurology, Ajou University School of Medicine, Suwon 442-749, South Korea. Tel.: +82 31219 5172; fax: +82 312195178.
} E-mail address: isjoo@ajou.ac.kr (I.S. Joo).

\section{Introduction}

Autoimmune myasthenia gravis (MG) frequently involves the extrinsic ocular muscles (EOM) and leads to the development of 
ptosis and/or diplopia. The disease clinically restricted to the EOM is referred to as ocular myasthenia gravis (OMG) [1]. Previous studies have reported that ocular disturbances are the first and sole manifestation of MG in about $50 \%$ of patients and that between $50 \%$ and $60 \%$ of patients presenting with OMG subsequently progress to develop generalized disease, usually within the first 1 to 2 years [2,3]. Early immunosuppressive therapy, specifically corticosteroids and/or azathioprine, has been suggested to prevent or delay the secondary generalization [4-8], but the cost-to-benefit ratio of this treatment remains controversial with no randomized controlled study conducted thus far [9]. Only a few studies have addressed the prognostic factors for OMG $[2,4]$, and several clinical and laboratory factors, such as older age at onset, presence of anti-acetylcholine receptor (AChR) antibody, high antibody titers, and the presence of thymoma, were claimed to be associated with an increased risk of secondary generalization.

The clinical expression of MG is believed to vary among populations, especially between Asians and Caucasians. For instance, in Asian populations, MG appears to have an earlier childhood onset with ocular disturbances alone when compared to Caucasian populations [10-13]. AChR antibody titers were also lower in the Chinese population of Taiwan than in Caucasians [12], and a relatively high prevalence of OMG was reported in Chinese adults living in Hong Kong [14]. In this study, we aimed to investigate the prevalence of OMG in a Korean MG patient population and its prognosis in terms of the development of secondary generalization. We also analyzed prognostic factors associated with the outcome, which would help us to select patients who are more likely to benefit from potentially risky treatments, such as the immunosuppressive agents and/or thymectomy.

\section{Patients and methods}

We conducted a retrospective multicenter survey in the year 2006. Investigators from a total of 13 medical college-affiliated tertiary hospitals, all of whom belonged to the Korean Research Group for Neuromuscular Diseases, participated in this study. All patients who were initially diagnosed with autoimmune MG at each center during the period from January 2000 to December 2005 were eligible for participation in the study. The diagnosis of autoimmune MG was made based on the results of conventional clinical or laboratory evaluations: exertional muscle weakness, positive Tensilon testing, AChR antibody, electrophysiological tests, including repetitive nerve stimulation test (RNST) and/or single fiber electromyography (SFEMG), and the exclusion of alternative diagnoses. The patients' medical records were reviewed by the local investigator at each center, and the data were extracted to fill in a uniform database (Microsoft ${ }^{\circledR}$ Office Access 2003) that was designed to include all clinical and laboratory data pertinent to the study.

Eligible patients were classified as having OMG or generalized MG (GMG) by the local investigator according to their clinical distribution of muscle involvement. In the study protocol and at a meeting of the investigators before the start of the study, much emphasis was placed on defining OMG as autoimmune MG 'clinically' limited to the levator palpabrae and/or extraocular muscles. Patients were classified as having generalized disease if there was any exertional weakness in the facial (except the orbicularis oculi), oropharyngeal, neck, respiratory, axial or limb muscles, either subjectively or during neurological examination. The results of electrophysiological tests in the facial and limb muscles were not criteria for the distinction between ocular and generalized diseases [15].

The diagnosis of ocular or generalized disease was made at two stages of the disease course: at symptom onset and at presentation. The time of symptom onset was defined as within the first month after onset. The follow-up duration refers to the time (months) from symptom presentation to the end of follow-ups, while the time from symptom onset to presentation was referred to as the initial disease duration. Based on this scheme, the patients with MG were categorized into four groups as follows: OMG at onset, GMG at onset, OMG at presentation, and GMG at presentation (Fig. 1). Since the data analysis was focused on the patients with OMG at presentation, we divided the patients in this group into two subgroups based on their prognosis with respect to the development of secondary generalization, i.e., the patients whose disease remained ocular throughout the follow-up duration (OMG-R) vs. the patients who progressed to develop generalized disease (OMG-G). In patients with OMG-G, secondary generalization was defined as the emergence of any symptoms and signs of exertional weakness characteristic of generalized disease during the follow-up duration. The results of AChR antibody, electrophysiological tests and chest computed tomography (CT) were obtained from the time of symptom presentation. Data on treatment modalities were included only if the treatment had been administered to the patients with OMG before they progressed to develop generalized disease. After data collection, patients were excluded from the analysis if they were younger than the age of 20 years, or when data were incomplete or duplicated in two or more centers.

The baseline characteristics were compared between the two subgroups (OMG-R and OMG-G) in order to investigate any factors

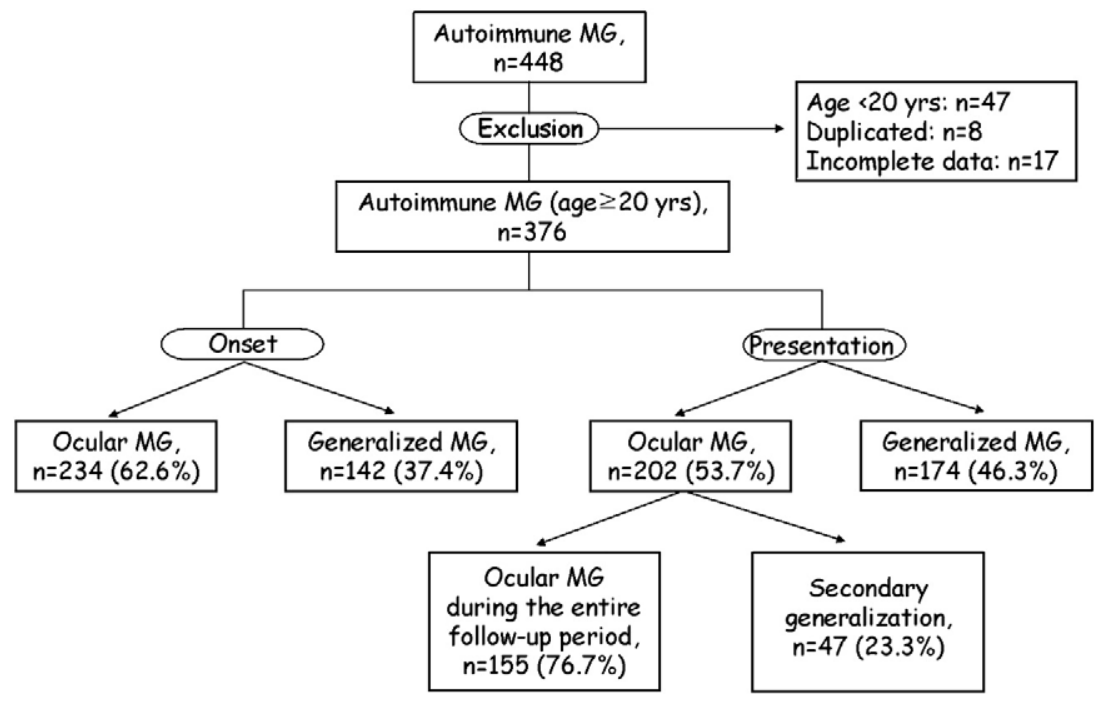

Fig. 1. Study profile. 
Table 1

Baseline characteristics of patients presenting with ocular myasthenia

\begin{tabular}{|c|c|c|c|c|}
\hline & \multicolumn{3}{|c|}{ Patients with OMG at presentation } & \multirow[t]{2}{*}{$p$ value } \\
\hline & Total $(n=202)$ & OMG-R $(n=155)$ & OMG-G $(n=47)$ & \\
\hline \multicolumn{4}{|l|}{ Sex } & \multirow[t]{3}{*}{ NS } \\
\hline Women & $114(56.4)$ & $89(57.4)$ & $25(53.2)$ & \\
\hline Men & $88(43.6)$ & $66(42.6)$ & $22(46.8)$ & \\
\hline \multicolumn{4}{|l|}{ Age at presentation } & \multirow[t]{3}{*}{ NS } \\
\hline$<50$ & $121(59.9)$ & $90(58.1)$ & $31(66.0)$ & \\
\hline$\geq 50$ & $81(40.1)$ & $65(41.9)$ & $16(44.0)$ & \\
\hline $\begin{array}{l}\text { Initial disease duration } \\
\text { (month) }\end{array}$ & $3(1-10)$ & $3(1-12)$ & $2(1-6)$ & $<0.05$ \\
\hline $\begin{array}{l}\text { Time to generalization }{ }^{\mathrm{a}} \\
\text { (month) }\end{array}$ & $8.9(3.0-27.9)$ & $11.8(5.0-34.3)$ & $3.0(1.0-9.0)$ & $<0.001$ \\
\hline \multicolumn{4}{|l|}{ AChR antibody } & \multirow[t]{3}{*}{$<0.001$} \\
\hline Seropositive & 135 (69.9) & $92(62.6)$ & $43(93.5)$ & \\
\hline Seronegative & $58(30.1)$ & $55(37.4)$ & $3(6.5)$ & \\
\hline AChR Antibody titer $(\mathrm{nM})^{\mathrm{b}}$ & $4.0(1.3-7.8)$ & $3.8(1.1-7.2)$ & $6.4(2.6-8.7)$ & $<0.05$ \\
\hline \multicolumn{5}{|c|}{ RNST $^{\mathrm{c}}$} \\
\hline Orbicularis oculi & $80 / 94(46.0)$ & $51 / 79(39.2)$ & $29 / 15(65.9)$ & $<0.01$ \\
\hline Trapezius & $40 / 47(46.0)$ & $25 / 40(38.5)$ & $15 / 7(68.2)$ & $<0.05$ \\
\hline Flexor carpi ulnaris & $14 / 42(25.0)$ & $7 / 34(17.1)$ & $7 / 8(46.7)$ & $<0.05^{*}$ \\
\hline Abductor digiti quinti & 28/158 (15.1) & $16 / 125(11.3)$ & $12 / 33(26.7)$ & $<0.05$ \\
\hline Any of the above mm & $100 / 89(52.9)$ & $68 / 75(47.6)$ & $32 / 14(69.6)$ & $<0.01$ \\
\hline \multicolumn{5}{|l|}{ Chest CT } \\
\hline Thymoma & $33(18.3)$ & $16(12.0)$ & $17(36.2)$ & $<0.001$ \\
\hline Thymic hyperplasia & $18(10.0)$ & $15(11.3)$ & $3(6.4)$ & NS \\
\hline Normal thymus & $129(71.7)$ & $102(76.7)$ & $27(57.4)$ & $<0.05$ \\
\hline Unchecked & 22 & 22 & & \\
\hline \multicolumn{5}{|l|}{$\begin{array}{l}\text { Immunomodulating } \\
\text { treatment }^{\mathrm{d}}\end{array}$} \\
\hline Thymectomy & $7(4.1)$ & $4(2.9)$ & $3(10.0)$ & NS* \\
\hline Prednisolone & $66(32.7)$ & $58(37.4)$ & $8(17.0)$ & $<0.01$ \\
\hline Other IS therapy & $26(12.9)$ & 20 (12.9) & $6(12.8)$ & NS \\
\hline
\end{tabular}

Values are $n$ (\%) or median (1st to 3rd quartiles).

OMG at presentation, ocular myasthenia gravis at the time of symptom presentation; OMG-R, patients with OMG at presentation whose disease remained ocular; OMG-G, patients with OMG at presentation who progressed to develop generalized MG; IS, immunosuppressive. *Fisher's exact test.

a The time from symptom presentation to the end of follow-ups in cases of OMG-R, and the time from symptom presentation to the development of secondary generalization in cases of OMG-G.

b Antibody titer in seropositive cases.

c Results are expressed as abnormal/normal (\%, proportion of abnormal results).

d In cases of OMG-G, treatments were included only if they were given before the development of secondary generalization.

e Thymectomy for nonthymomatous OMG.

associated with secondary generalization in OMG. Mann-Whitney $U$ and $\chi^{2}$ tests (or Fisher's exact test) were used as appropriate. Proportional hazards regression analyses were performed to evaluate the associations between various demographic and laboratory variables and the risk of secondary generalization throughout the followup duration. The multivariate model included age (years), sex, initial disease duration (months), and the other variables selected by the univariate analysis in which the probable variables with a $p$-value of no more than 0.1 were chosen for further analysis. Kaplan-Meier estimation was used to analyze the effect of early prednisolone treatment on the time to secondary generalization in patients with OMG after symptom presentation. $p<0.05$ was considered statistically significant. All data analyses were performed using SPSS version 11.0.1 (SPSS Inc., Chicago, Illinois).

\section{Results}

The well-documented medical records of 376 patients were evaluated for this retrospective analysis ( $\mathrm{M}: \mathrm{F}=152: 224$, mean age 45 years) (Fig. 1). Approximately half of the patients $(n==202,53.7 \%)$ presented with solely ocular disturbances, and the proportion of OMG was higher $(n=234,62.6 \%)$ at the time of symptom onset. Forty-seven patients (23.3\%) developed secondary generalization, mostly
(35 patients) within the first 6 months after symptom presentation (median 3 months), whereas the disease remained ocular throughout the follow-up duration (median 11.8 months) in the remaining 155 patients (76.7\%). The rate of secondary generalization was increased by approximately $10 \%$ (33.6\%, 39 of 116 patients) when the patients with less than 1 year of follow-up were excluded, whereas it was decreased by $14 \%$ (9.3\%, 8 of 86 patients) when only the patients who were followedup less than 1 year were analyzed. The baseline characteristics of the patients in these two subgroups (OMG-R vs. OMG-G) are summarized in Table 1 . There was no difference in sex and age at the time of symptom presentation between the two subgroups, but the initial disease duration was shorter in the patients in the OMG-G group than in those in the OMG-R group ( $p=0.04$, Mann-Whitney $U$ test).

The results of AChR antibody test at presentation were available in 193 patients. A total of 135 (70\%) patients tested positive for AChR antibodies $(\geq 0.2 \mathrm{nM})$, with titers ranging from 0.21 to $19.5 \mathrm{nM}$. The $A C h R$ antibody test was more frequently positive in the OMG-G group than in the OMG-R group $\left(p<0.001, \chi^{2}\right.$ test). In seropositive cases, its titer was also significantly higher in the OMG-G group than in the OMG-R group $(p=0.03$, Mann-Whitney $U$ test $)$. RNST was performed in the orbicularis oculi and/or proximal/distal limb muscles, the results of which were available in 189 patients. Abnormal decremental responses $(\geq 10 \%)$ were more frequently observed in the OMG-G group than in the OMG-R group in all of the muscles tested ( $p<0.01, \chi^{2}$ test). Chest CT was performed in 180 patients, and thymoma was detected in 33 patients (18.3\%). Thymoma was observed more frequently in the OMG-G group than in the OMG-R group (36.2\% vs. $12.0 \%, p<0.001, \chi^{2}$ test). A total of 192 patients (95\%) were treated with acetylcholinesterase inhibitors. An oral corticosteroid (prednisolone) was used for the treatment of OMG in 66 patients (32.7\%), more frequently in the OMG-R group than in the OMG$G$ group (37.4\% vs. $17.6 \%, p<0.01, \chi^{2}$ test). Azathioprine was combined with prednisolone in 22 patients, and used as a single immunosuppressive agent in 5 patients. Thymectomy was performed in 27 patients (13.4\%) with OMG at presentation, because of abnormal chest CT suggestive of thymoma. In cases without any evidence of thymoma on chest $C T$, the surgery was rarely performed in only 7 patients before the development of secondary generalization.

Cox proportional hazards regression analyses revealed that early oral prednisolone treatment significantly reduced the risk of secondary generalization ( $\mathrm{HR}, 0.24 ; 95 \% \mathrm{CI}, 0.11-0.56)$, whereas abnormal AChR antibody (HR, 5.34; 95\% CI, 1.60-17.8) and thymoma (HR, 2.32; $95 \%$ CI, 1.21-4.45) were predictive of the development of secondary generalization (Table 2). Kaplan-Meier estimates comparing the prednisolone-treated and -untreated patients are shown in Fig. 2.

Table 2

Univariate and multivariate proportional hazards regression analyses for secondary generalization

\begin{tabular}{|c|c|c|c|c|}
\hline \multirow[t]{2}{*}{ Variables } & \multicolumn{2}{|l|}{ Univariate } & \multicolumn{2}{|l|}{ Multivariate } \\
\hline & Hazard ratio $(95 \% \mathrm{CI})$ & $p$ value & Hazard ratio $(95 \% \mathrm{CI})$ & $p$ value \\
\hline Sex (women) & $0.78(0.43-1.41)$ & 0.41 & $0.70(0.36-1.35)$ & 0.28 \\
\hline Age ( $\geq 50$ years) & $0.80(0.43-1.48)$ & 0.47 & $0.80(0.43-1.51)$ & 0.50 \\
\hline $\begin{array}{l}\text { Time to presentation } \\
\text { ( } \geq 3 \text { months) }\end{array}$ & $0.64(0.35-1.16)$ & 0.14 & $0.87(0.47-1.64)$ & 0.68 \\
\hline $\begin{array}{l}\text { AChR antibody } \\
\text { (seropositive) }\end{array}$ & $6.29(1.95-20.3)$ & 0.002 & $5.34(1.60-17.8)$ & 0.006 \\
\hline RNST $^{\mathrm{a}}$ (abnormal) & $1.75(0.91-3.36)$ & 0.09 & $1.35(0.69-2.61)$ & 0.12 \\
\hline Thymoma $^{\mathrm{b}}$ & $3.26(1.77-6.01)$ & $<0.001$ & $2.32(1.21-4.46)$ & 0.01 \\
\hline $\begin{array}{l}\text { Prednisolone } \\
\text { treatment }^{c}\end{array}$ & $0.33(0.15-0.71)$ & 0.004 & $0.24(0.11-0.56)$ & 0.001 \\
\hline
\end{tabular}

\footnotetext{
a The results of RNST in any of the muscles tested (orbicularis oculi, trapezius, flexor carpi ulnaris, or abductor digiti quinti).

b Thymoma on chest computed tomography.

c Oral prednisolone treatment for ocular myasthenia, excluding the cases who received prednisolone treatment after the development of generalized disease.
} 


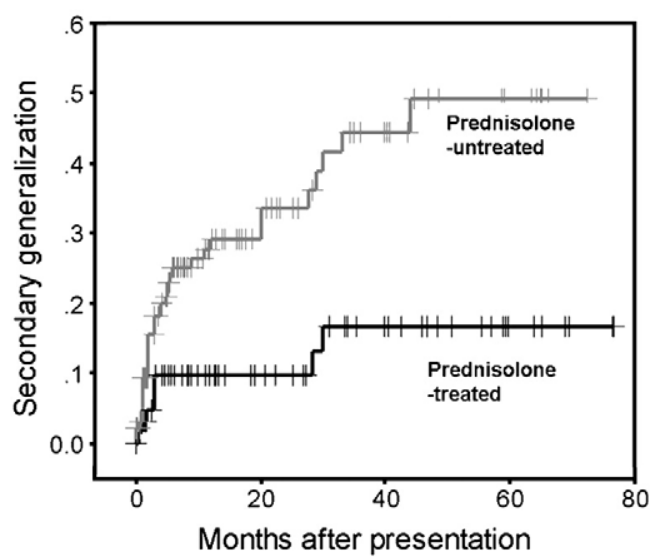

Fig. 2. Kaplan-Meier estimates for the cumulative development of secondary generalization after symptom presentation in prednisolone-treated or -untreated patients.

The log-rank test revealed a significant difference in the rate of secondary generalization between the two groups of patients.

\section{Discussion}

The current study is the largest retrospective analysis focused on OMG in an Asian adult population to our knowledge. This investigation was designed 1 ) to determine the prevalence of OMG and the rate of secondary generalization, and 2) to identify prognostic factors associated with secondary generalization in a Korean MG patient population. Our data are comparable in many respects to previous observations reported in Caucasians. First of all, the prevalence of OMG was very similar to those reported in earlier studies with approximately $50 \%$ of our patients presenting with solely ocular disturbances [3,4]. In addition, the current study adds to the increasing body of evidence that shows a beneficial effect of early corticosteroid treatment on the risk of secondary generalization [4-8]. Using proportional hazards regression model, this investigation also supports the conclusions of previously published data that the presence of abnormal AChR antibody and thymoma may be risk factors for the development of secondary generalization $[4,7]$.

Intriguingly, however, the prognosis of OMG seems to be much better in our patients with the rate of secondary generalization being approximately half of that reported in Caucasians [2,3]. This discrepancy may be related to the variable and short duration of follow-ups in the current retrospective study. Only about half the patients had been followed-up longer than 1 year in this investigation, which might have resulted in the underestimation of the rate. Indeed, when only these patients were analyzed, the rate turned out to be increased by around $10 \%$. Another explanation may be related to the rather long initial disease duration in our patients. The risk of secondary generalization is well known to have a strong inverse correlation with the duration of ocular disease [3,4,16-18]. Therefore, it is likely that the long initial disease duration in our patients could affect the subsequent generalization rate, i.e, a longer delay before presentation would imply a better prognosis. The patients who were at a higher risk of generalization during the early disease stages might have been excluded from the analysis on the secondary generalization rate which were analyzed only in the patients who were diagnosed as ocular MG at presentation. In fact, 32 (13.6\%) out of 234 patients who belonged to the group of ocular MG at onset were found to develop generalized disease before they sought medical attention, so that they were not eventually included in the analysis on secondary generalization rate. The influence of initial disease duration upon subsequent prognosis was also supported by the longer initial disease duration in patients whose disease remained ocular, compared to that in patients who progressed to develop generalized disease during follow-up periods. Finally, the low rate of secondary generalization could be explained by the early corticosteroid treatment which was given to about one-third of our patients. Indeed, the point estimates of hazard ratios in this investigation (0.24) were comparable to the figures $(0.19-0.32)$ reported in earlier studies $[4,7,8]$, supporting the hypothesis that early corticosteroid treatment can reduce the risk of generalization. However, it should be pointed out that the corticosteroid therapy may simply provide symptomatic control and mask overt evidence of generalization in patients on treatment. It also remains unclear whether the early corticosteroid therapy can truly prevent the occurrence of generalized disease or just delay its onset. Prospective studies should address these issues in the future.

Previously, investigators have set different criteria for the diagnosis of OMG with respect to the duration of disease. For example, Grob et al., included only the patients whose disease remained ocular for the first 1 month after onset [16], while Sommer et al., followed the criterion of Oosterhuis which required a period of at least 3 months after onset for the diagnosis of OMG $[3,4]$. Although these time limits were somewhat arbitrary, it was claimed that the occurrence of generalized disease during early disease stages would not qualify for the diagnosis of OMG as a separate entity [4]. Interestingly, Sommer et al., reported a much lower rate of secondary generalization (31\%) compared to that reported by Grob et al. (66\%) [4,16], and we suspect that part of the discrepancy may be related to the above difference in inclusion criteria. Unfortunately, there is no consensus to date on how long clinical manifestations should be restricted to the EOM in order to qualify for the diagnosis of OMG. Considering the above, in the current study we applied a two-step scheme for the classification of ocular vs. generalized disease, one at the onset (defined as within the first 1 month after onset) and the other at the time of presentation. We targeted on the status at presentation for analyzing the prognosis of OMG, because in our opinion it has at least three advantages. First, the diagnosis of OMG at presentation would be more reliable than that made at onset since the former was made based not only on historical information but also on the findings of neurological examination. Second, the classification at presentation would also be more relevant because any laboratory data having potentially prognostic implications, such as AChR antibody and electrophysiologic abnormalities, were available mostly at this time point. Finally, it can provide us information on the initial disease duration, which may have important prognostic implications in clinical practice as well as therapeutic trials for OMG.

Consistent with previous reports, the titers of AChR antibody were found to be low in patients with OMG in the current study $[12,18]$. However, the seropositivity rate (69.9\%) appears to be high in our patients compared to those $(40-70 \%)$ reported in Caucasians $[18,19]$. This issue needs to be addressed with respect to certain limitations probably inherent in a retrospective study like the current one. First, the diagnosis of OMG can be difficult in patients with atypical or subtle signs, particularly when diagnostic tests, such as AChR antibody assay and Tensilon testing, yield negative or equivocal results $[17,20]$. Therefore, we suspect that OMG may be underdiagnosed, particularly in seronegative and mild cases. Second, during the execution of this study, we became aware that categorization of the disease into two subgroups, ocular vs. generalized myasthenia, might not be as straightforward in reality as originally expected. This could be challenging particularly in the elderly patients who might complain of general weakness and nonspecific fatigue. Mild generalized MG could be misclassified into OMG or vice versa. We speculated that these two reasons might account for the high seropositivity rate of AChR antibody in this investigation. The same explanations can be given of the high frequency of thymoma (18.3\%) in our patients, although it was based on radiological findings and the data on histological confirmation were incomplete in the current study.

One of the interesting findings in our investigation is that abnormal results in RNST were more frequent in patients who 
progressed to develop generalized disease, although they were not predictive of secondary generalization. Surprisingly, only a few studies have evaluated the prognostic value of electrophysiological tests in patients with OMG. According to these observations, neither abnormal RNST nor SFEMG on limb muscles is predictive of secondary generalization, while normal SFEMG appears to have a negative predictive value $[2,4,21,22]$. In these studies, however, the number of patients and follow-up durations were too small and short to provide reliable data on this issue. Another concern might be the presence of a "grey area" between ocular and mild generalized disease, as mentioned earlier. The prognostic value of "latent" electrophysiological abnormalities could also have been complicated further by using the treatments possibly reducing the risk of secondary generalization. Future large-scale long-term prospective studies may shed light on this issue.

Taken at face value, the prevalence of OMG in our Korean MG patients appears to be similar to that in Caucasian patients, but the risk of secondary generalization seems to be lower in our patients. However, the results are complicated by the relatively short and variable duration of follow-ups, the long initial disease duration, and also by the use of corticosteroid therapy. For these reasons, we cannot draw any firm conclusions regarding ethnic differences in the prevalence and prognosis of OMG based on the results of this retrospective analysis. A long-term prospective observational study is warranted to properly address the issue. However, the data in this investigation implies that AChR antibody, thymoma, early corticosteroid treatment, and possibly "latent" neuromuscular transmission defects revealed by RNST may have impacts on the risk of developing generalized disease. Stratification with respect to these covariates should be considered in future randomized controlled trials of potentially effective treatments, and we propose that the role of early immunosuppressive therapy and thymectomy for OMG may differ between Asian and other populations who might have different risks for secondary generalization.

\section{Acknowledgment}

The authors are grateful to Seung Min Kim, MD, Chairman of the Korean Research Group for Neuromuscular Diseases (KRGND), as well as the other members of the KRGND for their advice and assistance with the study and preparation of this article.

\section{References}

[1] Jaretzki III A, Barohn RJ, Ernstoff RM, Kirkham TH, Knox DL, Miller NR, et al. Myasthenia gravis: recommendations for clinical research standards. Task Force of the Medical Scientific Advisory Board of the Myasthenia Gravis Foundation of America. Neurology 2000;55:16-23.

[2] Bever CT, Aquino AV, Penn AS, Lovelace RE, Rowland LP. Prognosis of ocular myasthenia. Ann Neurol 1983;14:516-9.

[3] Oosterhuis HJ. The natural course of myasthenia gravis: a longterm follow-up study. J Neurol Neurosurg Psychiatry 1989;52:1121-7.

[4] Sommer N, Sigg B, Melms A, Weller M, Schepelmann K, Herzau V, et al. Ocular myasthenia gravis: response to long-term immunosuppressive treatment. J Neurol Neurosurg Psychiatry 1997;62:156-62.

[5] Papapetropoulous TH, Ellul J, Tsibri E. Development of generalized myasthenia gravis in patients with ocular myasthenia. Arch Neurol 2003;60:1491-2.

[6] Mee J, Paine M, Byrne E, King J, Reardon K, O'Day J. Immunotherapy of ocular myasthenia gravis reduces conversion to generalized myasthenia gravis. J Neuroopthalmol 2003;23:251-5.

[7] Kupersmith MJ, Latkany R, Homel P. Development of generalized disease at 2 years in patients with ocular myasthenia gravis. Arch Neurol 2003;60:243-8.

[8] Monsul NT, Patwa HS, Knorr AM, Lesser RL, Goldstein JM. The effect of prednisone on the progression from ocular to generalized myasthenia gravis. J Neurol Sci 2004;217:131-3.

[9] Benatar M, Kaminski HJ. Evidence report: the medical treatment of ocular myasthenia (an evidence-based review): report of the Quality Standards Subcommittee of the American Academy of Neurology. Neurology 2007;68:2144-9.

[10] Uono M. Clinical statistics of myasthenia gravis in Japan. Int J Neurol 1980;14:87-99.

[11] Chan-Lui WY, Leung NK, Lau TTY. Myasthenia gravis in Chinese children. Dev Med Child Neurol 1984;26:717-24.

[12] Chiu HC, Vincent A, Newsom-Davis J, Hsieh KH, Hung TP. Myasthenia gravis: population differences in disease expression and acetylcholine receptor antibody titers between Chinese and Caucasians. Neurology 1987;37:1854-7.

[13] Lee KW, Kim JS, Lee SH, and MG Study Group. Epidemiological and clinical characteristics of myasthenia gravis in Korea. J Korean Neurol Assoc 1997;15:825-38.

[14] Yu YL, Hawkins BR, Ip MSM, Wong V, Woo E. Myasthenia gravis in Hong Kong Chinese. 1. Epidemiology and adult disease. Acta Neurol Scand 1992;86:113-9.

[15] Kupersmith MJ. Does early treatment of ocular myasthenia gravis with prednisone reduce progression to generalized disease? J Neurol Sci 2004;217:123-4.

[16] Grob D, Arsura EL, Brunner NG, Nambda T. The course of myasthenia gravis and therapies affecting outcome. Ann N Y Acad Sci 1987;505:472-99.

[17] Evoli A, Tonali P, Bartoccioni E, Lo Monaco M. Ocular myasthenia: diagnostic and therapeutic problems. Acta Neurol Scand 1988;77:31-5.

[18] Sommer N, Melms A, Weller M, Dichgans J. Ocular myasthenia gravis. A critical review of clinical and pathophysiological aspects. Doc Ophthalmol 1993;84:309-33.

[19] Vincent A, Newsom-Davis J. Acetylcholine receptor antibody as a diagnostic test for myasthenia gravis: results in 153 validated cases and 2967 diagnostic assays. J Neurol Neurosurg Psychiatry 1985;48:1246-52.

[20] Moorthy G, Behrens MM, Drachman DB, Kirkham TH, Knox DL, Miller NR, et al Ocular pseudomyasthenia or ocular myasthenia "plus": a warning to clinicians. Neurology 1989;39:1150-4.

[21] Thomas M, Ahuja GK, Behari M, Prasad K. Ocular myasthenia gravis-factors predictive for generalisation. J Assoc Phys India 1993;41:28-9.

[22] Weimberg DH, Rizzo JF, Hayes MT, Kneeland MD, Kelly JJ. Ocular myasthenia gravis: predictive value of single-fiber electromyography. Muscle Nerve 1999;22:1222-7. 